\title{
Leistungserbringung braucht öffentliche Sozialplanung
}

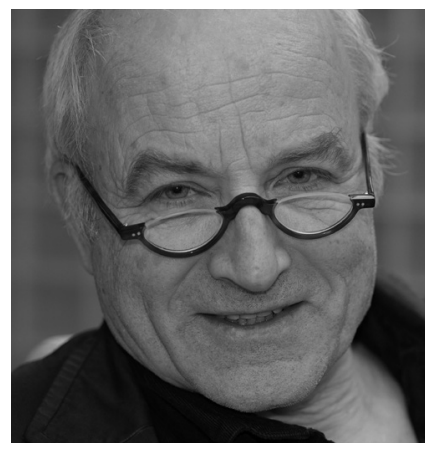

VON ANDREAS STRUNK Prof. Dr. phil. Andreas Strunk, Dipl. Ing. ist Sozialpädagoge und Sozialplaner. In der Gesellschaft für Innovation, Systementwicklung und Soziale Arbeit (GISAmbH) leitet er Projekte der Organisationsentwicklung und Sozialforschung. Weiterbildungen als Supervisor, lösungsorientierter Berater und Case Manager. Er ist Vorstandsmitglied im Deutschen Berufsverband für Soziale Arbeit (DBSH), Landesverband Baden-Württemberg. prof.strunk@t-online.de

\author{
Öffentliche Sozialplanung muss im Hinblick auf \\ die Erbringung sozialer Leistungen dafür sorgen, \\ dass Menschen ihre gesetzlichen Ansprüche erfüllt \\ bekommen. Dazu müssen Planungsprozesse organisiert \\ und mit den Beteiligten abgesprochen werden.
}

In jüngster Zeit wird wieder verstärkt über Möglichkeiten und Grenzen von öffentlicher Sozialplanung nachgedacht und Entsprechendes publiziert. Drei Hinweise mögen das verdeutlichen:

- Das Ministerium für Arbeit, Integration und Soziales des Landes Nordrhein-Westfalen hat ein Handbuch für Kommunen mit dem Titel »Moderne Sozialplanung « herausgegeben (Düsseldorf 2011); www.mais.nrw.de.

- Die Liga der Freien Wohlfahrtspflege in Thüringen erarbeitete mit Unterstützung des zuständigen Landesministeriums eine »Machbarkeitsstudie zur Implementierung eines strategischen Zentrums für Sozialplanung « (Erfurt 2012); www.liga-thueringen.de.

- Auf Fortbildungstagen wird zunehmend die Bedeutung guter Sozialplanung betont und dargestellt. Beispiel: »Sozialplanung - Gute Beispiele der Zusammenarbeit von Kommunen und Kreisen mit der Freien Wohlfahrtspflege « am 16. November 2012 in Düsseldorf.

Für diese Aktivitäten mag es verschiedene Gründe geben. Beispielsweise: die zunehmende gesellschaftliche Spaltung, das selektive Marktverhalten in Teilen der Sozialwirtschaft und die langsame Aushöhlung des Subsidiaritätsprinzips. (1) Auf eine weitergehende Ursachenanalyse wird hier verzichtet. Es soll vielmehr der Versuch unternommen werden, mit einigen methodologischen Überlegungen Gesichtspunkte zu formulieren, die für den Bereich der Leistungserbringung wichtig sind und
Gegenstand von Sozialplanung werden können.

Da das gesamte Feld der Sozialplanung komplex und wenig übersichtlich ist, empfiehlt es sich zum Hilfsmittel der Modellierung zu greifen, um eine hypothetische Ordnung zu schaffen, in der relevante Gesichtspunkte ins Blickfeld geraten können. Auf diese Weise entstehen heuristische Modelle. Dazu bieten sich zunächst zwei Visualisierungen an: »Die vier Wirkungskreise von Sozialplanung « (vgl. Abb. 1) und »Sozialplanung im Dreieck der Sozialwirtschaft« (vgl. Abb. 2).

Betrachten wir zunächst die erste Abbildung. Hier wurde der Versuch unternommen, öffentliche Sozialplanung in die Logik der Gewährung von Sozialleistungen einzuordnen, die im Prinzip für alle Sozialgesetzbücher gemäß SGB I gilt, denn dort ist folgende Aussage zu finden: »Die Leistungsträger sind verpflichtet, darauf hinzuwirken, dass jeder Berechtigte die ihm zustehenden Sozialleistungen in zeitgemäßer Weise, umfassend und zügig erhält und dass die zur Ausführung von Sozialleistungen erforderlichen Dienste und Einrichtungen rechtzeitig und ausreichend zur Verfügung stehen.«( $\mathbb{S} 17$, Abs. 1 SGB I).

Diese normativen Aussagen sind in der Praxis nur dann einzulösen, wenn die Kostenträger je nach Zuständigkeit (örtlich und/oder überörtlich) Zentren öffentlicher Sozialplanung schaffen, ausstatten und deren Arbeitsergebnisse verbindlich beachten. Die Zentren gestalten ihre Praxis jeweils nach der dargestellten Zirkularität. Mit dem Begriff »Zentrum« ist keine Größenordnung 


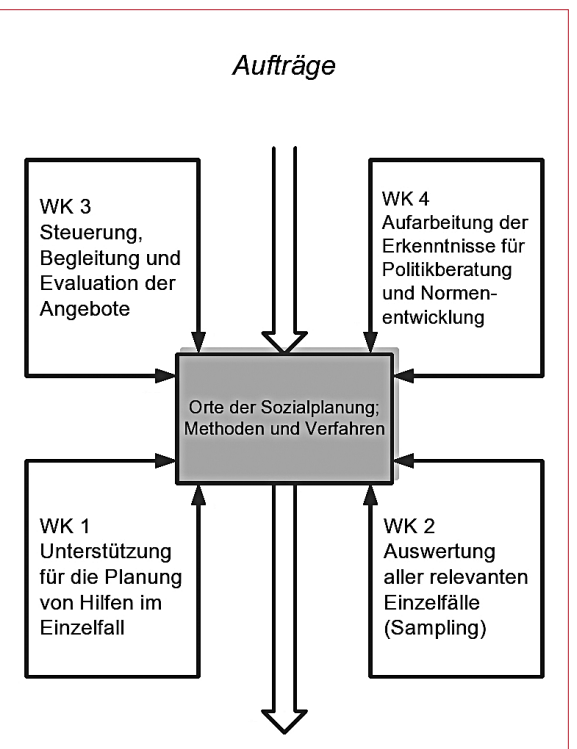

Ergebnisse

Abb. 1: Öffentliche Sozialplanung muss dafür sorgen, dass jeder Berechtigte "die ihm zustehenden Sozialleistungen in zeitgemäßer Weise, umfassend und zügig erhält und dass die zur Ausführung von Sozialleistungen erforderlichen Dienste und Einrichtungen rechtzeitig und ausreichend zur Verfügung stehen « (SGBI), wie beispielhaft an Wirkungskreisen (WK) gezeigt werden kann.

gemeint. Er soll nur darauf hinweisen, dass die öffentlichen Kostenträger gut beraten sind, entsprechende Arbeitseinheiten so zu konzipieren, dass sie qualitativ und quantitativ eine professionell gute Arbeit leisten können, jeweils bezogen auf das räumliche Gebiet, für das der Kostenträger zuständig ist. (2)

Bei der Arbeit der Sozialplanungszentren sind wichtige Schnittstellen zu beachten; beispielsweise zur kommunalen Planung (normiert von der jeweiligen Gemeindeordnung) und zu anderen öffentlichen Sozialplanungszentren (z. B. die der Behindertenhilfe). Im Prinzip ist eine integrierte Sozialplanung zu entwickeln, die eine horizontale Vernetzung (z. B. zur Flächennutzungsplanung) und eine vertikale Vernetzung (z. B. zur regionalen Planung des öffentlichen Nahverkehrs) betreibt. Im Rahmen einer integrierten Sozialplanung können auch Möglichkeiten und Grenzen von Kooperationen in der Leistungserbringung untersucht und ausgehandelt werden.

Um die Zirkularität öffentlicher Sozialplanung deutlicher werden $\mathrm{zu}$ lassen, werden beispielhaft vier Aufgaben innerhalb der Wirkungskreise angesprochen:
- Standardisierung von Hilfeplanverfahren zur Einzelfallhilfe, damit entsprechende Verläufe überhaupt ausgewertet werden können

- Festlegung von Kriterien für eine Sammlung und eine Auswertungsmethode, die sowohl quantitative als auch qualitative Merkmale bei der Evaluation einer ausreichenden Zahl von Einzelfällen zulässt

- Entwicklung, Implementation und Evaluation von fehlenden Angeboten, die notwendig geworden sind aufgrund der ausgewerteten Einzelfälle

- Ausarbeitung von Sozialberichten und Vorlagen für relevante parlamentarische Gremien

Nun findet öffentliche Sozialplanung in einem komplex-vernetzten System statt, das ansatzweise mit Hilfe der zweiten Abbildung zur »Sozialplanung im Dreieck der Sozialwirtschaft « visualisiert werden soll (vgl. Abb. 2). Diese Modellierung kann mit dem vorliegenden Text nicht ausführlich genug behandelt werden. Einige Aspekte werden aber auch ohne Kommentar deutlich. (3)

\section{Resümee}

Folgende vier Hinweise hinsichtlich der Funktion von öffentlicher Sozialplanung sind mir wichtig.

Erstens: Auch im Bereich der Sozialwirtschaft sind Wirkungen von Lobbyismus auf die politische Programmierung von Sozial- und Gesundheitspolitik deutlich geworden. (4) Öffentliche Sozialplanung kann diesen Einfluss setzt das eine Marktdynamik voraus, der nicht alle Leistungsberechtigten gewachsen sind und auch künftig nicht gewachsen sein werden. Öffentliche Sozialplanung kann hier ein differenzierteres und zutreffenderes Bild zeichnen, das u. a. auf Effekte des »Creaming the poor", das Konzentrieren auf schnelle Erfolge versprechende Zielgruppen, aufmerksam macht und Menschen schützt vor ihrer drohenden Zurichtung durch Anbieter der Sozialwirtschaft. In diesem Zusammenhang sei an die polemische Aussage von Klaus Dörner erinnert: »Heimträger sind Geiselnehmer und die Kostenträger leisten Beihilfe zur Geiselnahme«. (5)

Drittens: Unternehmen der Sozialwirtschaft werden zunehmend als »hybride Organisationen « beschrieben. (6) Gemeint ist u. a. der Sachverhalt, dass diese Unternehmen einen schwierigen Balanceakt zu bewältigen haben durch die Platzierung bedarfsgerechter Angebote im Spannungsfeld von Staat, Markt und Zivilgesellschaft. Öffentliche Sozialplanung steht vor einer ähnlichen Aufgabe. Sie muss auch Aussagen machen über gelingendes Leben von Leistungsberechtigten im konkreten Lebens- und Sozialraum der betroffenen Menschen. In diesem befinden sich glücklicherweise nicht nur Anbieter von Leistungen, die nach unterschiedlichen Sozialgesetzbüchern finanziert werden. Es geht zunehmend um die Beschreibung und Integration von Ressourcen, die als komplementär und subsidiär zu beschreiben sind. Die Forderung nach Lebens- und Sozialraumorientierung hat

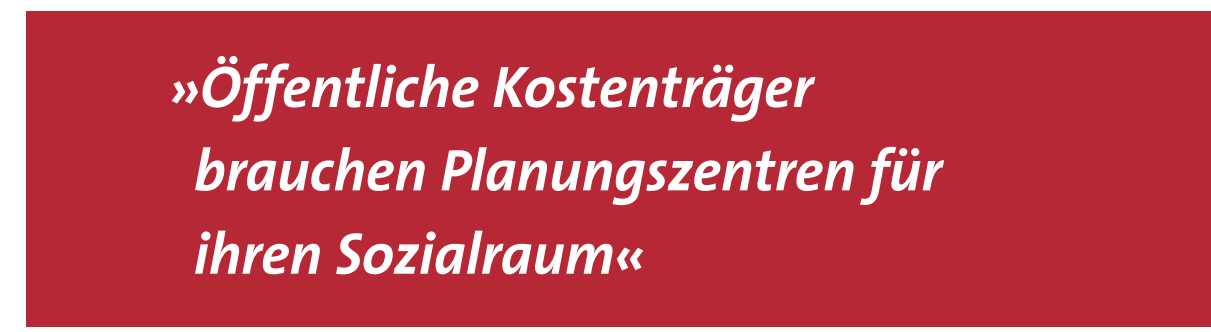

"zähmen« durch entsprechende Berichterstattungen, sofern diese in den parlamentarischen Kontexten beachtet werden.

Zweitens: Die zunehmende und teilweise verzerrende Beschreibung des Systems von Sozialer Arbeit als »Markt « setzt mit Recht auf die Kompetenzen von nachfragenden Menschen (Kaufkraft, Urteilskraft, Planungsvermögen und Rechtskenntnisse). Allerdings erhebliche Konsequenzen auf die Gestaltung von Kooperationen im Bereich der Leistungserbringung.

Viertens: Deutlich wird das an einer neuen Aufgabe für öffentliche Sozialplanung, die als Folge der UN-Behindertenrechtskonvention entstanden ist und die als »örtliche Teilhabeplanung « beschrieben wird. (7) Kooperationen in der Leistungserbringung sind dann $\mathrm{zu}$ bewerten und einzuordnen in einem 


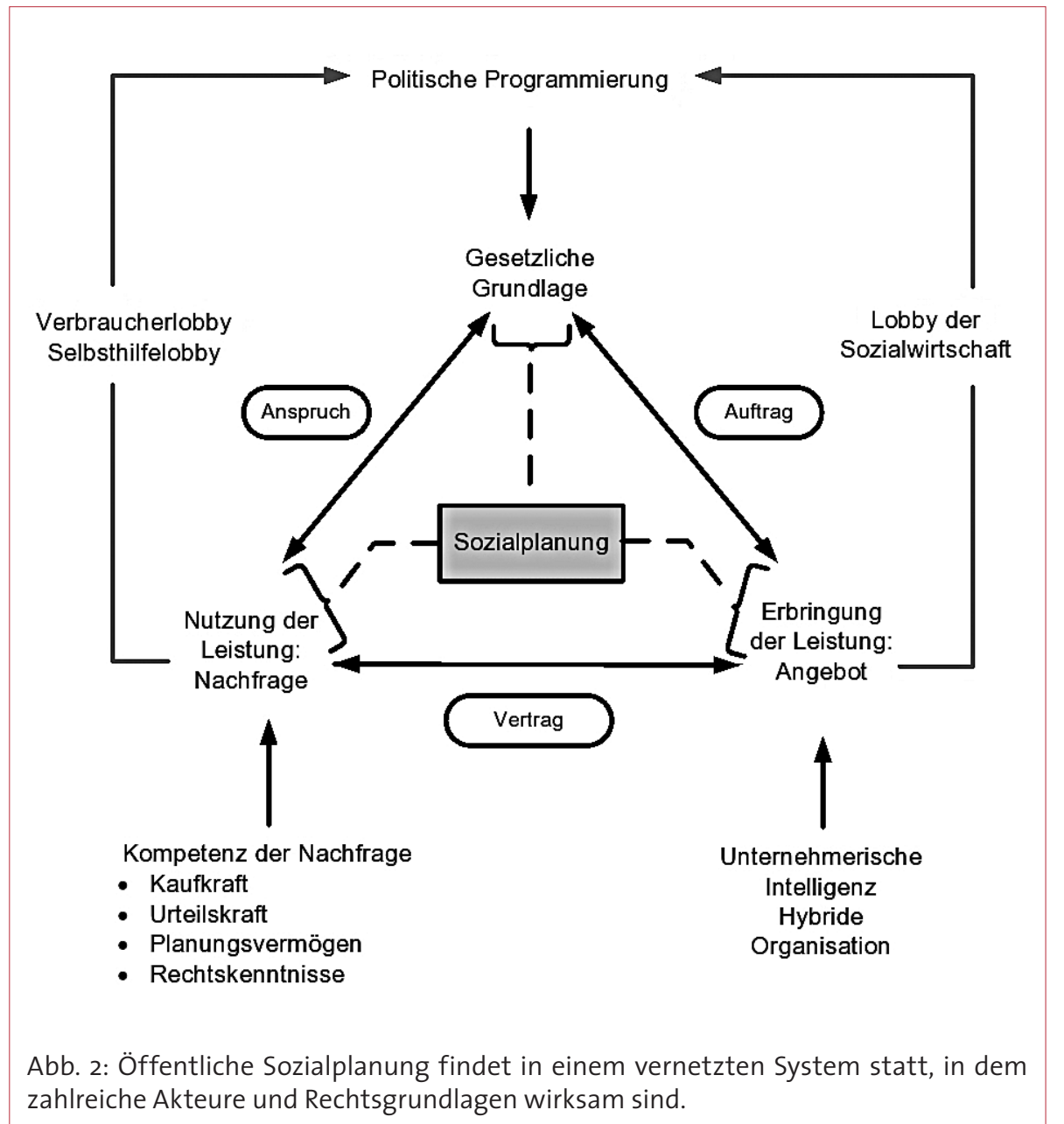

Konzept des Designs für alle, denn was beispielsweise für Behinderte gut ist, ist gut für Alte, und was für Behinderte und Alte gut ist, ist für uns alle gut.

\section{Anmerkungen}

(1) Vgl. dazu folgende Einschätzung: "Zunehmend wird die Meinung laut, die freie Wohlfahrt sei Dienstleisterin statt Mitgestalterin des Sozialen."So die Referentin Rotraud Kießling im Diakonischen Werk Sachsen in: wohnungslos 3 (2012), S. 89.

(2) Vgl. dazu: Verein für Sozialplanung e. V. (VSOP), Kompass Sozialplanung - Zwischen Gestaltung und Verwaltung im Reformprozess, Speyer 2006.

(3) Weiterführende Überlegungen finden sich bei: Andreas Strunk, Die Sehnsucht nach einer Alternative wächst, in: FORUMsozial 1 (2010), S. $22-26$.

(4) Vgl. dazu: Extreme Dummheit. Der sozialdemokratische Gesundheitsexperte Karl Lauterbach beschreibt den Druck mächtiger Lobbygruppen auf Abgeordnete und Regierung, in Der Spiegel, S. 22 f.

(5) Vgl. dazu: Klaus Dörner, Heimträger sind Geiselnehmer, in: Caritas NRW 5 (2001).

(6) Vgl. dazu: Wolfgang Wasel und Hanns-Stephan Haas, Hybride Organisationen. Antworten auf Markt und Inklusion, in: Nachrichtendienst des Deutschen Vereins für öffentliche und private Fürsorge 12 (2012), S. 586 - 593.

(7) Vgl. dazu: Andreas Strunk, Die inklusive Gemeinde. Eine Vision für gelingendes Leben vor Ort, in: Blätter der Wohlfahrtspflege 4 (2012), S. $146-149$.
Verfall des Humanen durch organisationales Handeln

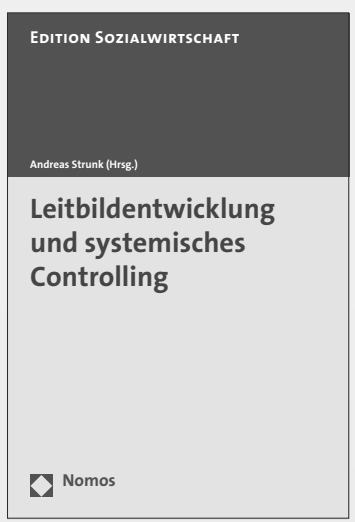

Leitbildentwicklung und systemisches Controlling Herausgegeben von Prof. Dr. Andreas Strunk

2013, 232 S., brosch., 29,- $€$

ISBN 978-3-8329-6473-3

(Edition Sozialwirtschaft, Bd. 35)

Verfasst von renommierten Autoren aus Wissenschaft und Praxis, richtet sich der Band an Führungskräfte der Sozialwirtschaft, die sich im Hinblick auf eigene Leitbildentwicklungen orientieren wollen, Beraterinnen und Berater, die Vorhaben der Leitbildentwicklung konzipieren sowie Fachleute aus Wissenschaft und Ausbildung, die bestehende Modelle evaluieren wollen.

www.nomos-shop.de/13492

Nomos 